dietary supplements are available both as combination formulas and as singleingredient supplements.

The most studies, investigating diet influence on RMD symptoms in human, were conducted among Rheumatoid Arthritis (RA) patients as an adjunctive therapy. Further, there is a wide variation in evidence robustness probably due to the complexity of studying the relationship between diet and disease activity.

Diets: Several controlled studies among RA patients have been performed.

Mediterranean diet intervention studies have shown tendency to pain reduction and improvement of physical function after 3-6 months.

An intervention study, comparing 7-10 days fasting followed by 13 months vegetarian diet and the ordinary diet, showed significant pain reduction in the intervention group. Though, there was no significant difference in physical function or morning stiffness compared to RA patients adhered to an ordinary diet.

Vegan diet intervention studies did not report statistical significant difference in pain, physical activity or morning stiffness compared to an ordinary diet.

One study compared 6 weeks of elimination diet to an ordinary diet. Due to inadequate data reporting, no between-group analyses were possible, the authors of the study concluded: "When the dietary and placebo groups were compared the dietary group did better for all 13 variables for which differences between them were significant".

Cholesterol lowering diet study among 17 Systemic Lupus Erythematosus (SLE) patients showed increased quality of life (measured by questionnaire) after 12 weeks study period compared to the control group.

Ramadan fasting study among 40 SLE patients did not reveal any influence of fasting on disease activity or patients' quality of life during the fasting period or 3 months after fasting compared to non-fasting SLE patients.

Non-randomised controlled low-salt, uncooked vegan diet study among 53 fibromyalgia patients revealed improvements in pain, joint stiffness, quality of sleep, quality of life and general health after 3 months of study period.

Dietary supplements: Several studies of fish oil supplementation have been performed among RA patients and have generally shown positive results on pain reduction, morning stiffness, and improvement in physical activity and decreased use of pain relief medications. The potential benefit of eating whole foods with high omega-3 content has not been evaluated.

Studies, investigating Vitamin D supplementation in RA patients did not find any disease modifying effect.

Interventional studies of antioxidant supplementation in patients with RMD have been inconclusive.

Current data regarding potential therapeutic effects of probiotics suggest plausible benefits, though evidence grade is still low.

There is some evidence that herbal therapy containing Gama Linolenic Acid oils (evening primrose, borage, or blackcurrant seed oil) reduce some RA symptoms.

Alcohol: Studying effects of alcohol on RMD activity is complicated not least in relation to the treatment. Two observational studies among RA patients, showed tendency towards an inverse association between alcohol use and disease severity.

Conclusion: The effects of dietary manipulation in RMD patients are still uncertain due to small study samples and potential risk of bias. Higher drop-out rates and weight loss in the groups with manipulated diets indicate that potential adverse events should not be ignored.

However, there is some evidence that fasting followed by a vegetarian diet and Mediterranean diet improve pain, but not stiffness and physical function among RA patients, when compared to an ordinary diet.

Several controlled studies showed that dietary supplements of moderate-to-high doses of omega- 3 fatty acids have a beneficial effect on several parameters of RA activity.

Evidence regarding diet influence on RMD's other than RA is very weak.

Disclosure of Interest: None declared

DOI: 10.1136/annrheumdis-2018-eular.7773

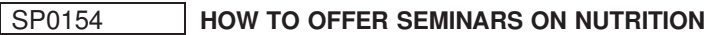

C. Elling-Audersch. Deutsche Rheuma-Liga (German Rheumatism League),

Berlin, Germany

Deutsche Rheuma-Liga (German Rheumatism League)

Deutsche Rheuma-Liga (DRL) is a patient organisation that offers support to people with all rheumatic and musculoskeletal diseases (RMDs). We offer to our members information, various seminars, exercise classes and advice services. The main goal is to improve a patient's self-management.

Most patients have questions concerning medication, but would like to know as well what other measures can be taken to improve their health. Nutrition and the question of a rheumatism diet is one of the great patients' demands.
Based on a survey of our organisation (DRL) patients' information priorities are obvious and have led to offerings for patients.

Our seminars for patients about nutrition are based on our nutrition booklet, on evidence-based information and on avoiding an esoteric content.

All seminars deal with three key elements of self-management: nutrition, exercise and ergonomic kitchen aids.

Based on the key messages of nutrition, the important aspects are not only to educate, but also to exercise, to communicate, to exchange experiences, to cook, to taste, to smell and to enjoy. We show ergonomic kitchen aids and explain their daily usefulness.

My talk will consider organisational aspects as well, if an organisation wants to offer such seminars.

In our experience combining nutrition, exercise and ergonomic aids are essential aspects of a healthy life and contribute to a person's rheumatic and/or musculoskeletal treatment successfully.

Disclosure of Interest: None declared

DOI: 10.1136/annrheumdis-2018-eular.7730

\section{SP0155 NUTRITION - HOW TO TALK ABOUT IT}

\section{Krafft. Rheumaliga Schweiz, Zurich, Switzerland}

When it comes to the subject of nutrition and rheumatism, particularly in relation to inflammatory rheumatism, opinions are sharply divided. Holistically oriented physicians and therapists believe that a change in diet can replace medication in the treatment of chronic inflammation and they thus pursue the goal of reducing the need for medication through an anti-inflammatory diet. Rheumatologists by contrast staunchly defend drug therapy and still approach the subject of nutrition with great reserve, disinterest, or even reject its effects out of hand. Some warn that patients who stray from a balanced diet with a mix of foods may risk nutritional deficiencies.

These opposing views leave many of those affected by rheumatism unsure of how to approach nutrition. Can a change in diet really help alleviate the symptoms of inflammatory rheumatism? Which diet rules can I trust? And what's more: How do I speak about it with my rheumatologist? Will he advocate a change in diet and view it as part of my arthritis therapy - or see it merely as a supporting measure, quite a nice idea but without any therapeutic value?

Anyone wanting to provide rheumatism patients with some guidance in this regard must ward against possible bias. The Swiss League against Rheumatism makes sure its communications on this controversial issue take into account the whole spectrum of existing medical pluralism. On the question of nutritional benefits, it thus provides information from the point of view of conventional medicine as well as that of complementary medicine and all the shades in between.

Objectives of the Swiss League against Rheumatism

The Swiss League against Rheumatism endeavours on the one hand to ensure that its communication about nutrition is not one-sided but rather presents the different viewpoints. On the other hand, those affected should be given access to knowledge that can help them to form their own opinion.

To achieve these goals, the Swiss League against Rheumatism continually invests human and financial resources into research on this topic, interviews and exchanges with experts, as well as ongoing updates of their contributions.

Communication channels: The Swiss League against Rheumatism informs people about the topic of nutrition through the following communication channels:

- Website

- Facebook

- Brochures

- Lectures

The main focus is on articles on the website.

Standpoint of the Swiss League against Rheumatism

The Swiss League against Rheumatism includes the subject of nutrition in its communications because there is a great need for information among those affected.

The Swiss League against Rheumatism itself takes no position for or against a described measure but rather only makes recommendations based on scientific criteria. On the basis of these scientific criteria, it can be assumed that nutrition, in particular a Mediterranean diet, can have a "positive influence" on various rheumatic diseases

Disclosure of Interest: None declared

DOI: 10.1136/annrheumdis-2018-eular.7850 